\title{
Combined treatment with niclosamide ethanolamine and artemether combination improves type 1 diabetes via the targeting of liver mitochondria
}

\author{
WENCI WENG ${ }^{1 *}$, HONGHONG LIU $^{2 *}$, ZHIJIAN SUN ${ }^{1}$, PENG ZHOU $^{1}$, XUEWEN YU $^{3}$, \\ MUMIN SHAO $^{3}$, PENGXUN HAN ${ }^{1}$ and HUILI SUN ${ }^{1}$ \\ ${ }^{1}$ Department of Nephrology, Shenzhen Traditional Chinese Medicine Hospital,
}

The Fourth Clinical Medical College of Guangzhou University of Chinese Medicine; ${ }^{2}$ Department of Nephrology,

Shenzhen Traditional Chinese Medicine Hospital Affiliated to Nanjing University of Chinese Medicine;

${ }^{3}$ Department of Pathology, Shenzhen Traditional Chinese Medicine Hospital, The Fourth Clinical Medical College of

Guangzhou University of Chinese Medicine, Shenzhen, Guangdong 518033, P.R. China

Received October 18, 2021; Accepted January 6, 2022

DOI: $10.3892 / \mathrm{etm} .2022 .11164$

\begin{abstract}
Type 1 diabetes (T1D) is characterized by dysregulated blood glucose and liver metabolism. In previous studies, niclosamide ethanolamine salt (NEN) and artemether (Art) displayed significant hypoglycemic effects. However, their combined therapeutic effects on the liver in T1D have remained elusive. In the present study, T1D mice were established and randomly allocated into groups. Following treatment, the physiological and metabolic parameters, including liver function, glycogen content, glucose-6-phosphatase (G6Pase) protein expression levels, mitochondrial biogenesis and mitochondrial metabolism were analyzed. Compared with the NEN or Art treatments alone, their combination improved glycometabolism and the symptoms of diabetes. Combined treatment with NEN and Art also significantly ameliorated liver injury and increased liver glycogen storage. Furthermore, combinatorial treatment significantly downregulated hepatic G6Pase protein expression levels and regulated mitochondrial biogenesis. NEN and Art increased the respiratory exchange rate and reduced mitochondrial phosphoenolpyruvate carboxykinase and branched-chain $\alpha$-keto acid dehydrogenase complex protein expression levels, whereby the effects were obviously
\end{abstract}

Correspondence to: Professor Huili Sun or Dr Pengxun Han, Department of Nephrology, Shenzhen Traditional Chinese Medicine Hospital, The Fourth Clinical Medical College of Guangzhou University of Chinese Medicine, 1 Fuhua Road, Futian, Shenzhen, Guangdong 518033, P.R. China

E-mail: sunhuili2011@126.com

E-mail: hanpengxun@126.com

*Contributed equally

Key words: niclosamide ethanolamine, artemether, type 1 diabetes, liver, mitochondria enhanced by their application as a combined treatment. In conclusion, the present study confirmed that combined treatment with NEN and Art improved glycometabolism and liver function in T1D mice and the therapeutic effects may be partially associated with the regulation of liver mitochondria.

\section{Introduction}

Type 1 diabetes (T1D) is characterized by the destruction of pancreatic $\beta$-cells, which results in insulin deficiency. The etiology and pathogenesis of the disease remain to be fully elucidated. Insulin replacement is currently the only treatment for T1D (1).

Blood glucose is tightly maintained within its normal range by numerous factors that affect glucose production and utilization. The key hormones involved in blood glucose regulation mainly include insulin, glucagon, epinephrine, glucocorticoid and growth hormone (2). As the primary organ of biochemical metabolism, the liver serves an important role in glucose homeostasis (3). Net hepatic glucose production is the summation of gluconeogenesis, glycogenolysis, glycogen synthesis and glycolysis, among other metabolic pathways (4). Glycogen is a multibranched polysaccharide of glucose, whereby liver glycogen is involved in the regulation of blood glucose homeostasis. Under normal physiological conditions, glycogen is synthesized following ingestion and broken down during fasting periods. However, dysregulated glycogen metabolism is observed in the diabetic liver (5). Furthermore, other metabolic pathways display abnormalities to varying degrees as a result of T1D (6).

Mitochondria are the main organelles for substance and energy metabolism. Hepatic mitochondrial dysfunction serves an important role in excessive hepatic glucose production and impaired glucose utilization $(7,8)$. Previous studies have demonstrated that niclosamide ethanolamine salt (NEN) and artemether (Art) displayed significant hypoglycemic effects in diabetes (9-12). Their pharmacological actions are closely related to the regulation of mitochondrial function $(13,14)$. 
However, their combined therapeutic effects on the liver in T1D have remained elusive.

In the present study, the hepatoprotective effects of combined NEN and Art treatment in T1D mice and the potential underlying mechanisms were investigated.

\section{Materials and methods}

Animal experiment. A total of 60 male C57BL/6 mice (body weight, 22-26 g, 8 weeks) were purchased from Guangdong Medical Laboratory Animal Center and were housed in the Central Animal Facility at Shenzhen Graduate School of Peking University (Shenzhen, China). The temperature was controlled at $20-23^{\circ} \mathrm{C}$, the relative humidity was controlled at $50-60 \%$, the light and dark cycle was $12 / 12 \mathrm{~h}$. All mice had free access to water and food. The diabetic mice were randomly allocated to the following groups ( $n=8$ per group): T1D group (T1D), T1D+NEN group (NEN), T1D+Art group (Art) and T1D+NEN+Art group (NEN+Art). T1D was established by administering multiple intraperitoneal injections of streptozotocin (STZ; $55 \mathrm{mg} / \mathrm{kg}$ ) to mice on five consecutive days. Control mice $(n=8)$ were intraperitoneally injected with the same volume of the vehicle. Successful induction of T1D was confirmed according to fasting blood glucose levels of $\geq 11.1 \mathrm{mmol} / \mathrm{l}$ at 9 days after the last injection of STZ. Mice in the control and T1D groups were fed a conventional standard diet; Mice in the NEN group were fed a standard diet containing $10 \mathrm{~g} / \mathrm{kg} \mathrm{NEN}$; mice in the Art group were fed a standard diet containing $0.67 \mathrm{~g} / \mathrm{kg}$ Art; and mice in the NEN+Art group were fed a standard diet containing $10 \mathrm{~g} / \mathrm{kg}$ NEN and $0.67 \mathrm{~g} / \mathrm{kg}$ Art. The diet was freely available to each group mice in their cage. The doses of NEN and Art used in the present study were selected according to previous studies $(9,12)$. The treatment lasted for 8 weeks. NEN was purchased from Hubei ShengTian HengChuang Biological Technology Co., Ltd. and Art was purchased from Chengdu ConBon Biotech Co., Ltd. All animal procedures were approved by the Guangzhou University of Chinese Medicine Institutional Animal Care and Use Committee (Shenzhen, China).

Tissue preparation. The body weight of the animals in each group was determined at the end of the study. The mice were anesthetized with $\sim 1 \%$ isoflurane and euthanized by cervical dislocation. Blood samples and liver tissues were rapidly collected and the livers were weighed. Partial liver tissues were fixed in $10 \%$ formalin and used for histopathological examination and immunohistochemical staining. The remaining liver tissues were immediately snap-frozen in liquid nitrogen and stored at $-80^{\circ} \mathrm{C}$ for later analysis.

Physiological and metabolic parameters. Fasting blood glucose was analyzed using a blood glucose meter (Roche Diagnostics). Urine was collected using metabolic cages (Tecniplast). Glycated hemoglobin (HbA1c) levels were analyzed using an Ultra2 HbA1c Analyzer (Primus). Serum alanine transaminase (ALT), aspartate transaminase (AST), total protein (TP), albumin (ALB), triglyceride (TG), total cholesterol (TC) and urinary glucose were detected using an automatic biochemical analyzer (Roche Diagnostics). Liver glycogen was quantified using the Anthrone method (15). Prior to the end of the experiment, the respiratory exchange ratio (RER) of the mice was determined using the Comprehensive Lab Animal Monitoring System (CLAMS; Columbus Instruments). The limb grip strength was measured using a dynamometer (ZH-YLS-13A; Anhui Zhenghua Biological Instrument Equipment Co., Ltd.) according to the manufacturer's instructions. Three measurements were performed and the mean value was calculated.

Light microscopy. From each specimen, three to six liver sections were randomly selected and stained with periodic acid-Schiff (PAS) and diastase (D)-PAS (Beijing Solarbio Science \& Technology Co., Ltd.) according to the manufacturer's protocols. The sections were scanned using the Digital Slide Scanner (3DHistech Ltd.) to evaluate the liver glycogen content.

ELISA. Serum insulin levels were determined using an ELISA kit (EMD Millipore; cat. no. \#EZRMI-13K) according to the manufacturer's protocol.

Immunohistochemical staining. Liver sections (3-6 slices) were deparaffinized and rehydrated. Subsequently, the sections were subjected to antigen retrieval by boiling in citric acid buffer ( $\mathrm{pH}$ 6) for $20 \mathrm{~min}$. After rinsing three times with PBS, the sections were incubated with primary antibodies against glucose-6-phosphatase (G6Pase; Abcam, ab83690, 1:100) overnight at $4^{\circ} \mathrm{C}$. After washing with PBS, the sections were incubated with HRP-polymer conjugated anti-mouse/rabbit IgG secondary antibody for $1 \mathrm{~h}$ at room temperature. Diaminobenzidine solution was used as a chromogen. The sections were counterstained with hematoxylin and images were acquired using the Digital Slide Scanner (3DHistech Ltd.).

Immunoblotting analysis. Liver tissues were homogenized and prepared in sample loading buffer (Bio-Rad Laboratories, Inc.). Total protein was separated using 10\% SDS-PAGE and transferred onto polyvinylidene difluoride membranes (EMD Millipore). After blocking with 5\% non-fat dry milk (Bio-Rad Laboratories, Inc.), the membranes were incubated at $4^{\circ} \mathrm{C}$ overnight with the following primary antibodies: G6Pase (Abcam, ab83690, 1:1,000), transcription factor A mitochondrial (TFAM; EMD Millipore, ABE483, 1:1,000), cytochrome $c$ oxidase IV (COX IV), translocase of the outer mitochondrial membrane 20 (TOM20), voltage-dependent anion channel (VDAC), pyruvate dehydrogenase (PDH), phosphoenolpyruvate carboxykinase (PCK2), branched-chain $\alpha$-keto acid dehydrogenase complex (BCKDH) and $\beta$-actin. COX IV (cat. no. \#4844, 1:1,000), TOM20 (\#13929, 1:1,000), VDAC (\#4661, 1:1,000), PDH (\#2784, 1:1,000), PCK2 (\#6924, 1:1,000) and BCKDH (\#90198, 1:1,000) antibodies were purchased from Cell Signaling Technology, Inc. and $\beta$-actin antibody was from MilliporeSigma (A5441, 1:2,000). The membranes were then incubated with HRP-conjugated Goat anti-Rabbit or Goat anti-Mouse secondary antibodies (Invitrogen; Thermo Fisher Scientific, Inc.; Cat. nos. 65-6120 or 62-6520, respectively; all 1:2,000) and detected by the ChemiDoc ${ }^{\mathrm{TM}}$ MP Imaging System (Bio-Rad Laboratories, Inc.).

Statistical analysis. Values are expressed as the mean \pm standard deviation. Data analysis was performed using SPSS statistics software version 22.0 (IBM Corporation). One-way ANOVA followed by Bonferroni's post-hoc test was used for 
A
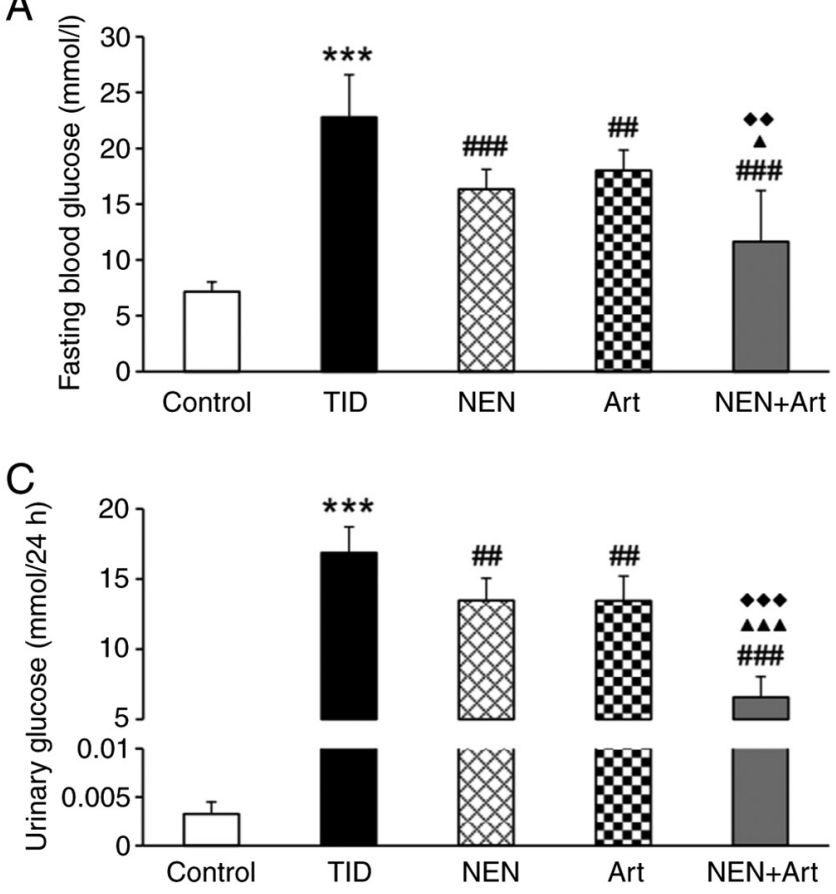

E

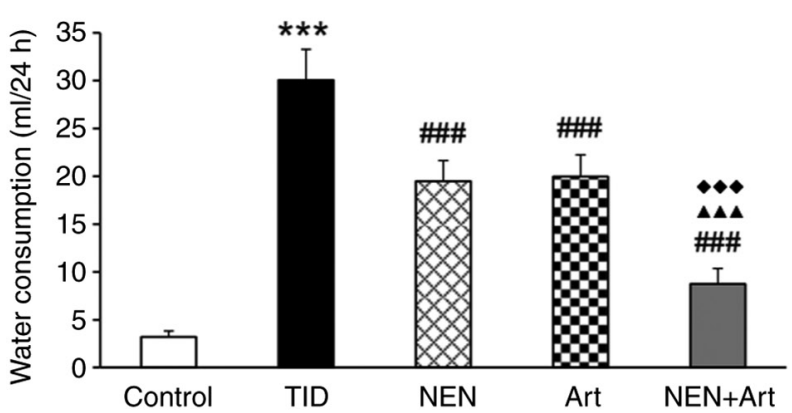

G

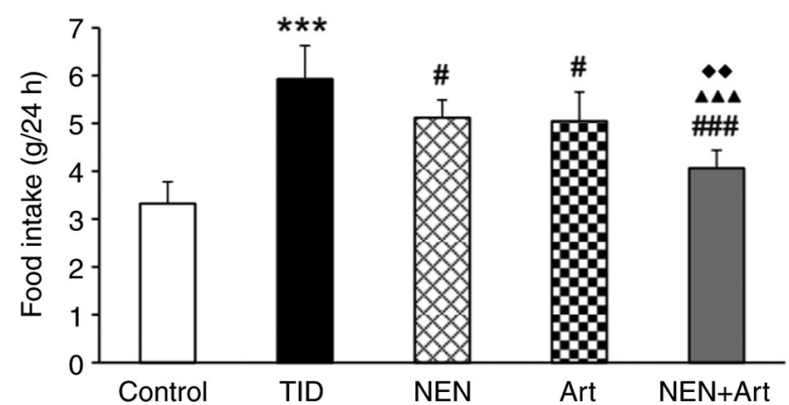

B

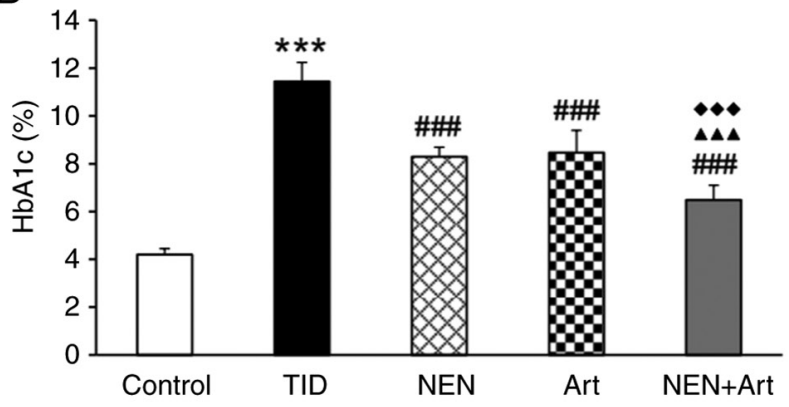

D

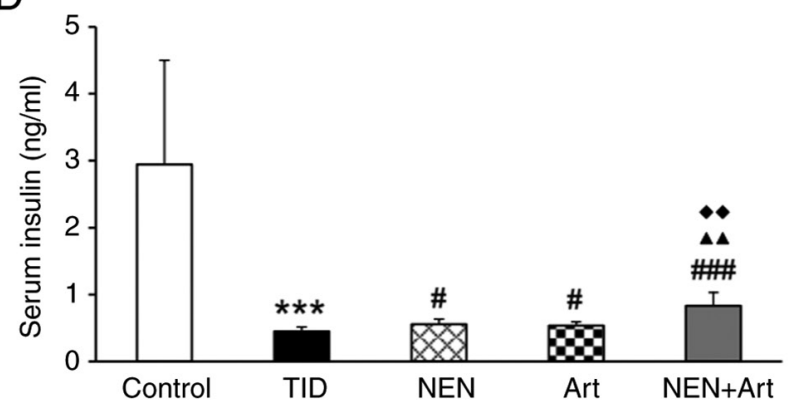

$\mathrm{F}$

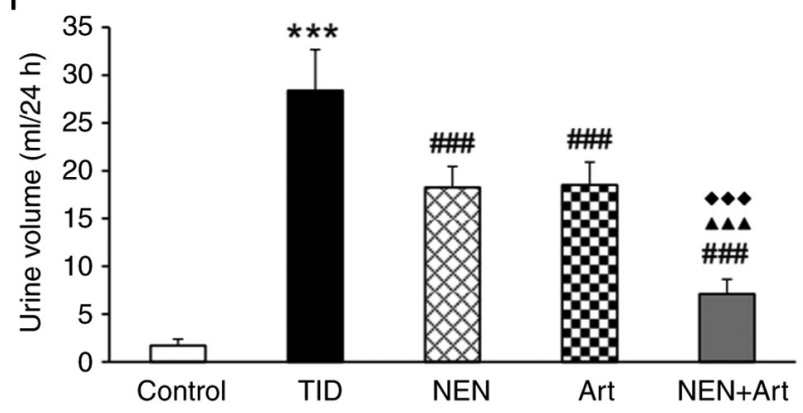

$\mathrm{H}$

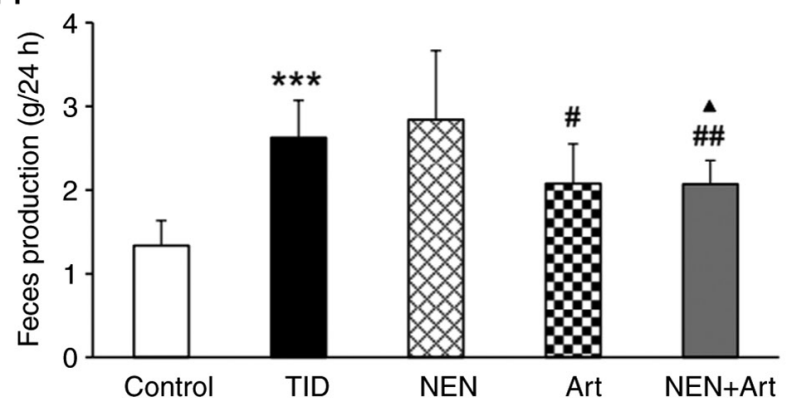

Figure 1. NEN+Art improves glycometabolism and T1D symptoms. (A-D) Changes in glycometabolism-related indices, including (A) fasting blood glucose, (B) HbAlc, (C) urinary glucose, and (D) serum insulin in the different groups after 8 weeks of treatment. (E) Water consumption, (F) urine volume, (G) food intake, and $(\mathrm{H})$ feces production after 8 weeks treatment in each group $\left(\mathrm{n}=8\right.$ per group). ${ }^{* * * *} \mathrm{P}<0.001$ vs. the control group; ${ }^{\#} \mathrm{P}<0.05$, ${ }^{\# \#} \mathrm{P}<0.01$ and ${ }^{\# \# \#} \mathrm{P}<0.001$ vs. the T1D group; ${ }^{\boldsymbol{\Delta}} \mathrm{P}<0.05,{ }^{\boldsymbol{\Delta}}{ }^{\mathrm{P}} \mathrm{P}<0.01$ and ${ }^{\boldsymbol{\Delta} \boldsymbol{\Delta} \boldsymbol{\Delta}} \mathrm{P}<0.001$ vs. the NEN group; ${ }^{\bullet} \mathrm{P}<0.01$ and ${ }^{\bullet}{ }^{*} \mathrm{P}<0.001$ vs. the Art group. NEN, niclosamide ethanolamine salt; Art, artemether; T1D, type 1 diabetes; HbAlc, glycated hemoglobin.

data analysis. $\mathrm{P}<0.05$ was considered to indicate a statistically significant difference.

\section{Results}

NEN+Art improves glycometabolism and diabetic symptoms. Compared with the control group, significantly increased fasting blood glucose, HbAlc and urinary glucose, and decreased serum insulin were observed in the T1D group mice (Fig. 1A-D). Consistent with a glycometabolic disorder, the mice in the T1D group exhibited typical diabetic symptoms including polydipsia, polyuria, polyphagia and increased feces production (Fig. 1E-H). These results confirmed that T1D in mice was well established and the success rate of establishing the model was $\sim 85 \%$. The combined treatment of NEN+Art reduced hyperglycemia and improved diabetic 

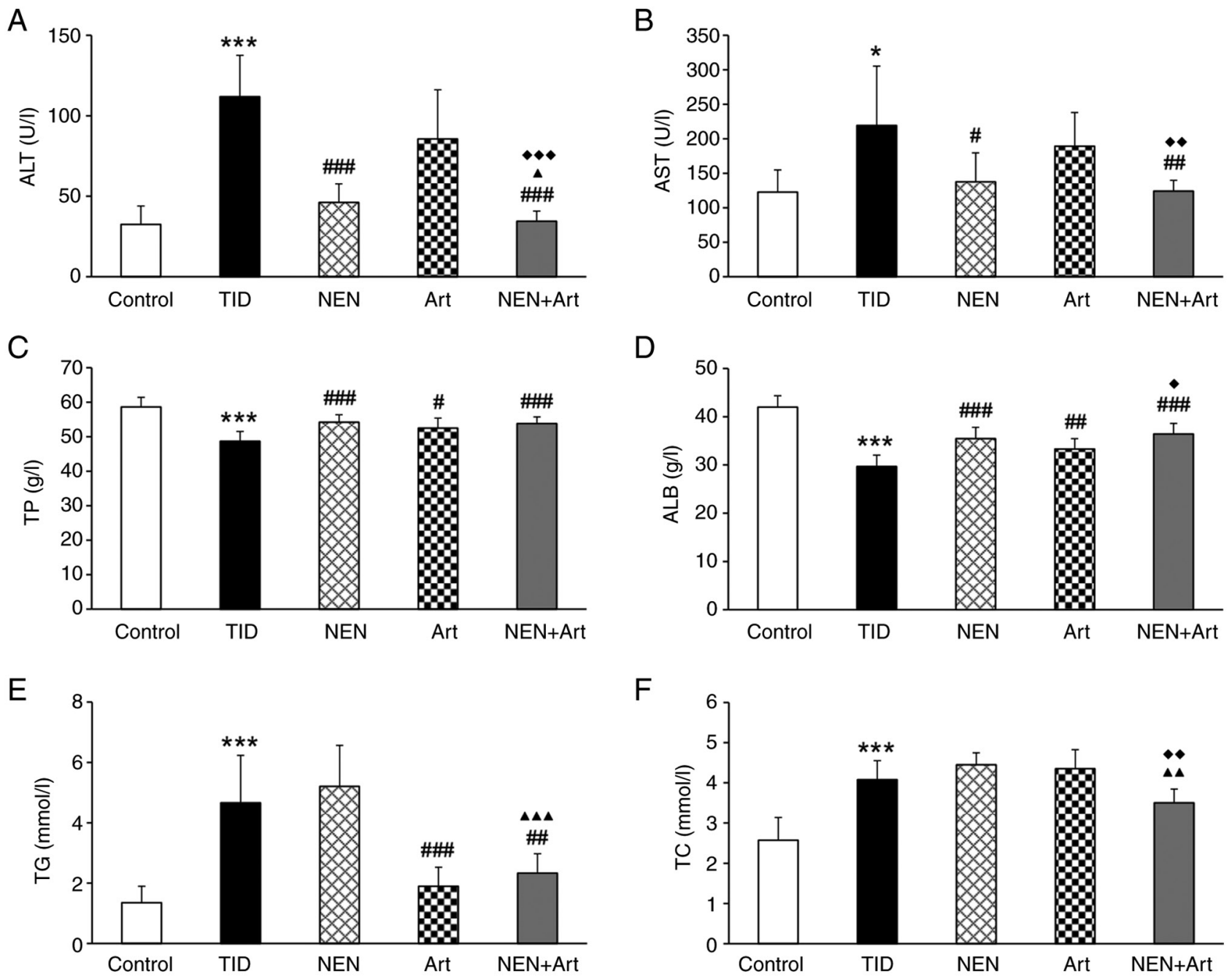

Figure 2. Effects of NEN, Art and NEN+Art on liver function and serum lipid. (A-F) Bar graphs display the serum levels of (A) ALT, (B) AST, (C) TP, (D) ALB, (E) TG, and (F) TC in each group at the end of the study ( $\mathrm{n}=8$ per group). ${ }^{*} \mathrm{P}<0.05$ and ${ }^{* * * *} \mathrm{P}<0.001$ vs. the control group; ${ }^{\#} \mathrm{P}<0.05$, ${ }^{\# \#} \mathrm{P}<0.01$ and ${ }^{\# \# \# /} \mathrm{P}<0.001 \mathrm{vs}$. the T1D group; ${ }^{\Delta} \mathrm{P}<0.05,{ }^{\Delta} \boldsymbol{P}<0.01$ and ${ }^{\Delta \Delta} \boldsymbol{\Delta} \mathrm{P}<0.001$ vs. the NEN group; ${ }^{\circ} \mathrm{P}<0.05,{ }^{\bullet} \mathrm{P}<0.01$ and ${ }^{\bullet}{ }^{\bullet} \mathrm{P}<0.001$ vs. the Art group. NEN, niclosamide ethanolamine salt; Art, artemether; T1D, type 1 diabetes; ALT, alanine transaminase; AST, aspartate transaminase; TP, total protein; ALB, albumin; TG, triglycerides; TC, total cholesterol.

symptoms to a greater extent than treatment with NEN or Art alone.

NEN+Art significantly ameliorates liver injury and reduces serum lipid levels. The liver serves important roles in glucose and lipid metabolism homeostasis. Compared to the control group, the T1D group exhibited elevated serum ALT, AST, TG and TC levels and decreased TP and ALB levels (Fig. 2). NEN treatment alone significantly reduced serum ALT and AST levels and increased TP and ALB levels, whereas TG and TC levels were increased to a certain extent but not to a significant degree. Art treatment alone significantly increased TP and ALB levels and decreased TG levels, but did not significantly affect ALT, AST and TC levels. Overall, NEN+Art more prominently ameliorated the T1D-associated aberrations in these liver function biomarkers.

$N E N+$ Art increases liver glycogen storage. Significantly increased liver weight and reduced liver glycogen were observed in the T1D group (Fig. 3A and B). NEN or Art treatment alone did not significantly increase the liver glycogen content. However, NEN+Art markedly increased the glycogen content (Fig. 3B). To further determine glycogen storage in the liver, PAS and D-PAS staining on liver sections was performed. The images demonstrated that glycogen changes in various groups were consistent with the biochemical measurement results (Fig. 3C and D).

Effects of NEN and Art on hepatic G6Pase protein expression levels. As presented in Fig. 4A and B, the immunoblotting results demonstrated that hepatic G6Pase protein expression levels significantly increased in T1D mice. NEN and Art treatment alone or as a combined therapy significantly downregulated G6Pase protein expression levels. Immunohistochemical staining for G6Pase also confirmed its protein expression trends in the different groups (Fig. 4C).

NEN and Art regulate hepatic mitochondrial biogenesis. Compared with the control group, TFAM protein expression levels increased significantly in the T1D group (Fig. 5A and B). Furthermore, mitochondrial-associated proteins, including VDAC, TOM20 and COX IV also increased to varying 
A

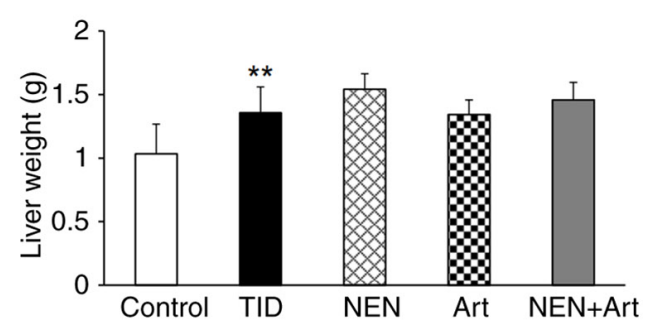

B

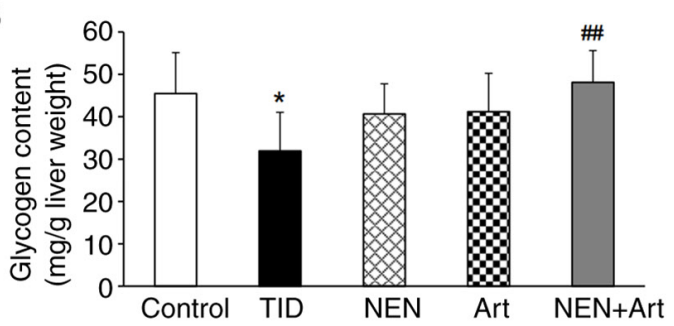

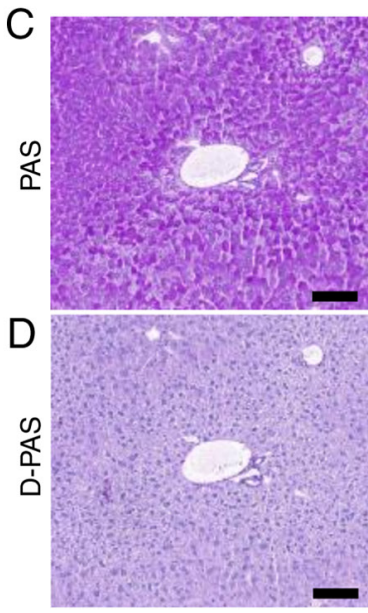

Control

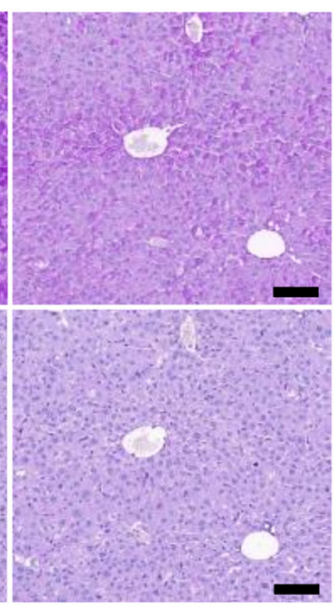

TID

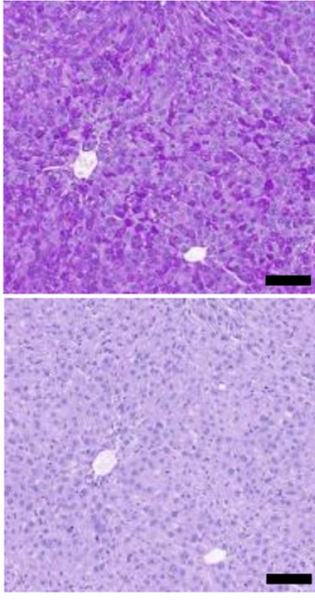

TID+NEN

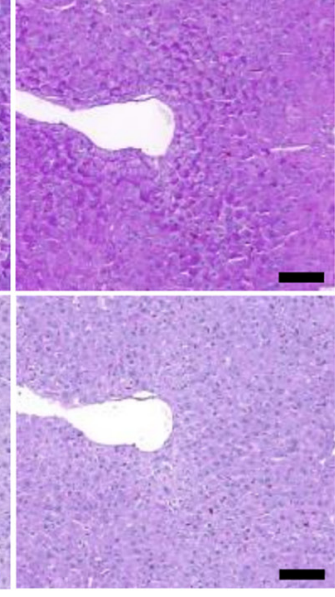

TID+Art

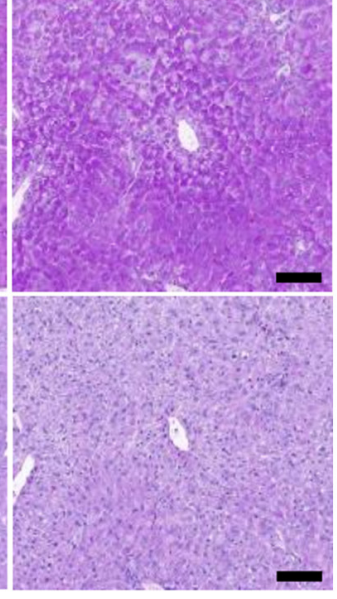

TID+NEN+Art

Figure 3. NEN+Art increases the liver glycogen content. (A and B) Bar graphs display (A) the liver weight and (B) liver glycogen content in each group (n=8 per group). ${ }^{*} \mathrm{P}<0.05$ and ${ }^{* *} \mathrm{P}<0.01$ vs. control group; ${ }^{\# \#} \mathrm{P}<0.01$ vs. the T1D group. (C and D) Representative images of (C) PAS and (D) D-PAS staining for liver tissues of the different groups (scale bar, $100 \mu \mathrm{m}$ ). NEN, niclosamide ethanolamine salt; Art, artemether; T1D, type 1 diabetes; D-PAS, diastase periodic acid Schiff.

A

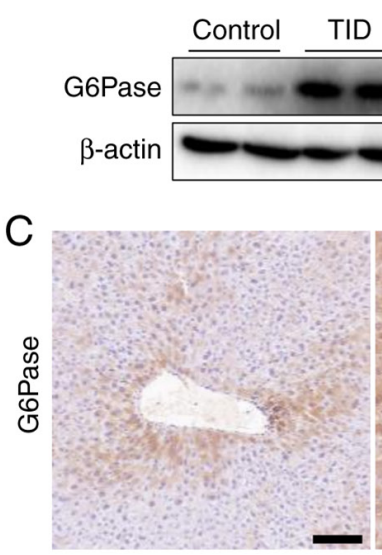

Control

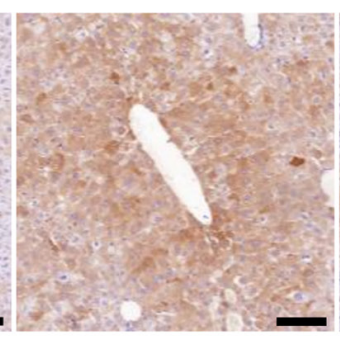

TID

\section{B}

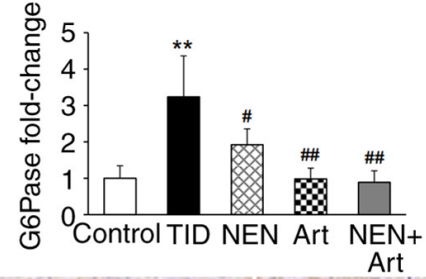

Figure 4. NEN and Art reduce the hepatic G6Pase protein expression levels. (A and B) Western blot analysis was performed to determine the hepatic protein expression levels of G6Pase in each group. (A) Western blot bands and (B) bar graphs displaying the fold change of G6Pase protein expression levels following normalization to $\beta$-actin ( $\mathrm{n}=4-6$ per group). ${ }^{* *} \mathrm{P}<0.01$ vs. the control group; ${ }^{\#} \mathrm{P}<0.05$ and ${ }^{\# \#} \mathrm{P}<0.01$ vs. the T1D group. (C) Representative immunostaining images of hepatic G6Pase in each group (scale bar, $100 \mu \mathrm{m}$ ). NEN, niclosamide ethanolamine salt; Art, artemether; T1D, type 1 diabetes; G6Pase, glucose-6-phosphatase.

degrees (Fig. 5C-E). NEN and Art both exerted suppressive effects on mitochondrial biogenesis and these effects were significantly enhanced when the treatments were combined.

$N E N+$ Art increases $R E R$ and regulates mitochondrial metabolism. Compared with the control group, the T1D group exhibited a significantly lower RER, which indicated that carbohydrates were not the predominant fuel source in the diabetic mice (Fig. 6A and B). During the dark (active) phase, either NEN or Art were able to raise the RER and this effect was enhanced in the group with the NEN+Art combined treatment. During the light (sleep) phase, only NEN and the NEN+Art combination were able to significantly raise the RER. As presented in Fig. 6C and D, there was no significant difference in PDH protein expression levels among the groups. Compared with the control mice, PCK2 and BCKDH 


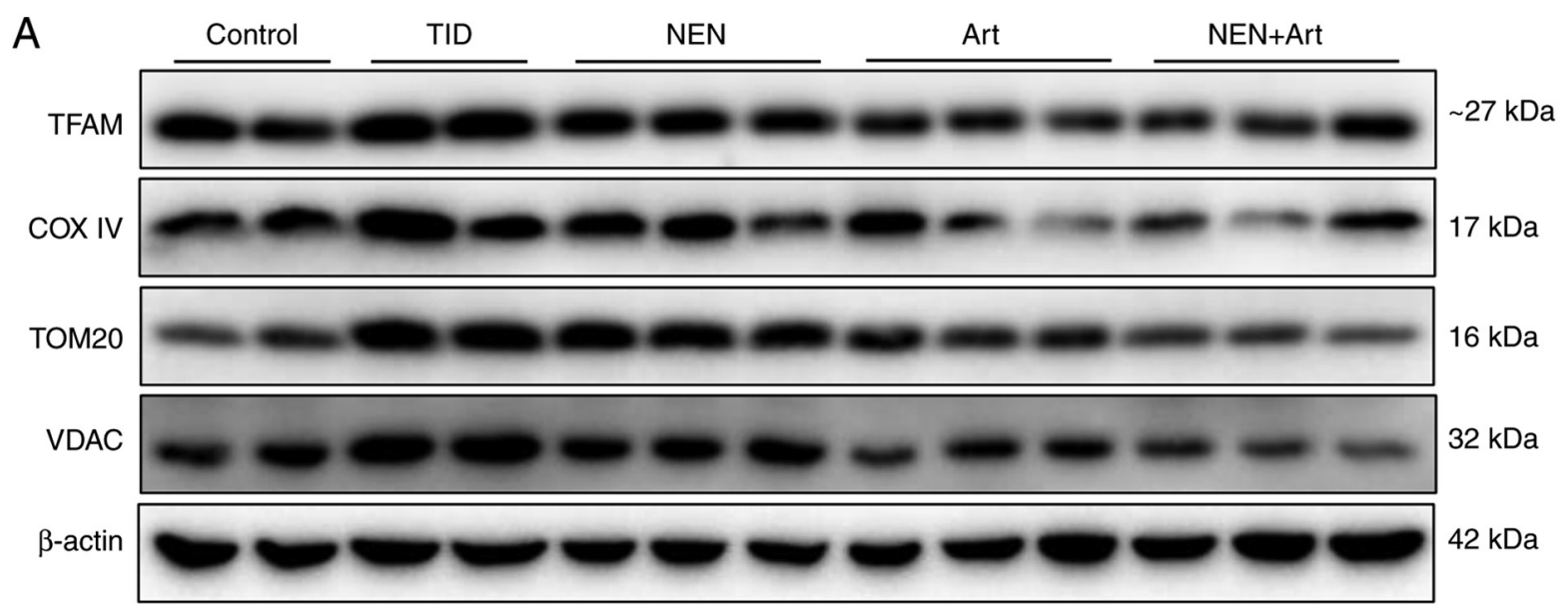

$\mathrm{B}$

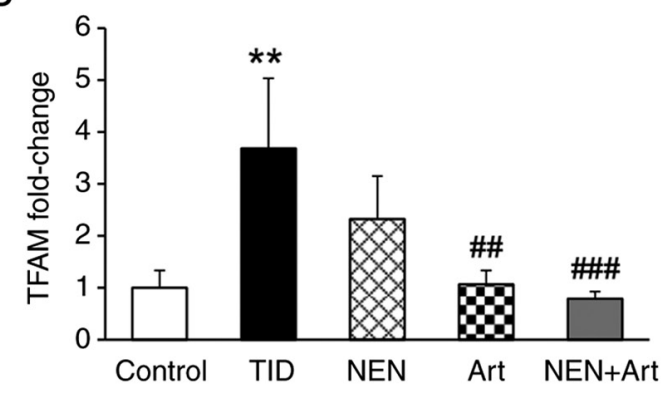

D

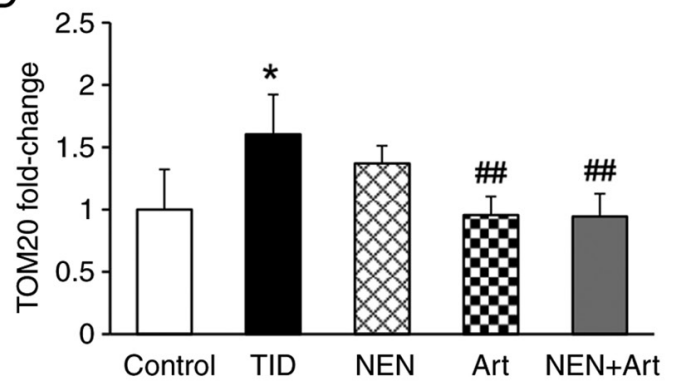

C

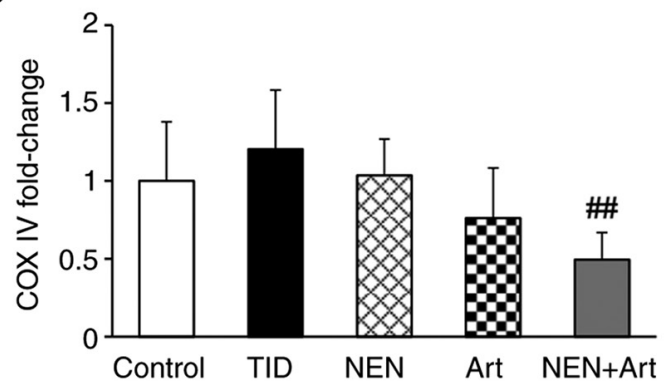

$E$

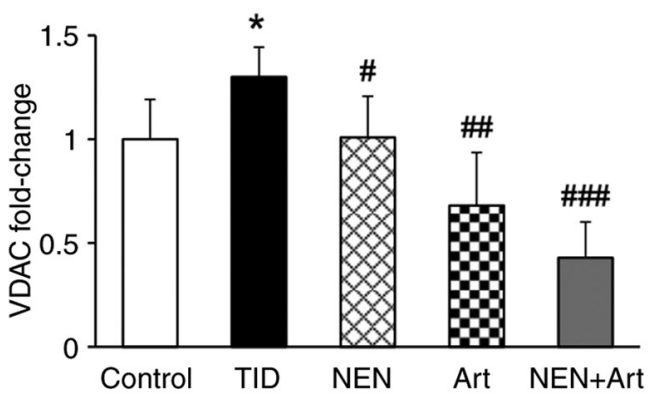

Figure 5. Effect of NEN and Art on hepatic mitochondrial biogenesis. Western blot analysis was performed to determine the protein expression levels of TFAM, COX IV, TOM20 and VDAC in the hepatic tissue of the different groups. (A) Western blot bands and (B-E) semi-quantitative analysis of (B) TFAM, (C) COX IV, (D) TOM20 and (E) VDAC protein expression levels in each group ( $\mathrm{n}=4-6$ per group). ${ }^{*} \mathrm{P}<0.05$ and ${ }^{* *} \mathrm{P}<0.01$ vs. the control group; ${ }^{\#} \mathrm{P}<0.05$, ${ }^{\# \#} \mathrm{P}<0.01$ and ${ }^{\# \# \#} \mathrm{P}<0.001$ vs. the T1D group. NEN, niclosamide ethanolamine salt; Art, artemether; T1D, type 1 diabetes; TFAM, transcription factor $\mathrm{A}$ mitochondrial; COX IV, cytochrome c oxidase IV; TOM20, translocase of the outer mitochondrial membrane 20; VDAC, voltage-dependent anion channel.

protein expression levels were significantly increased in T1D mice (Fig. 6E and F). NEN treatment alone downregulated PCK2 but did not influence BCKDH protein expression levels. Art treatment alone significantly downregulated both PCK2 and BCKDH protein expression levels. Similar to those in the Art group, the protein expression levels of PCK2 and $\mathrm{BCKDH}$ in the NEN+Art group were also significantly reduced.

Effects of NEN and Art on body weight and limb grip strength. In order to explore the contribution of muscle function on the RER in T1D, the body weight and limb grip strength in each group of mice were measured. As presented in Fig. 7, significantly reduced body weight and limb grip strength were observed in the T1D group when compared with those in the control group. These results may suggest that the contribution of muscle metabolism on the RER was weakened in the T1D group. NEN and NEN+Art treatment significantly increased the body weight, but only NEN treatment enhanced limb grip strength. Art treatment did not significantly affect the body weight and limb grip strength.

\section{Discussion}

In the present study, it was demonstrated that a combined treatment approach with NEN and Art more effectively improved T1D symptoms and glycometabolism compared with either treatment administered separately. The therapeutic effects may be related to the regulation of hepatic glycogen metabolism and mitochondrial function.

Consistent with previous studies, NEN or Art treatment alone displayed a hypoglycemic effect; however, the two treatments do not necessarily use the same mechanism (9-12). The therapeutic effect was significantly enhanced in the NEN+Art 
A

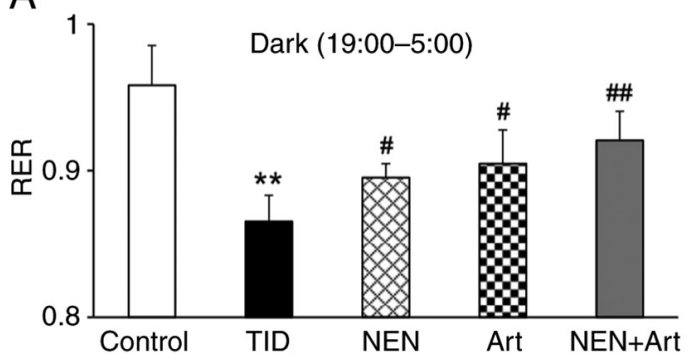

B

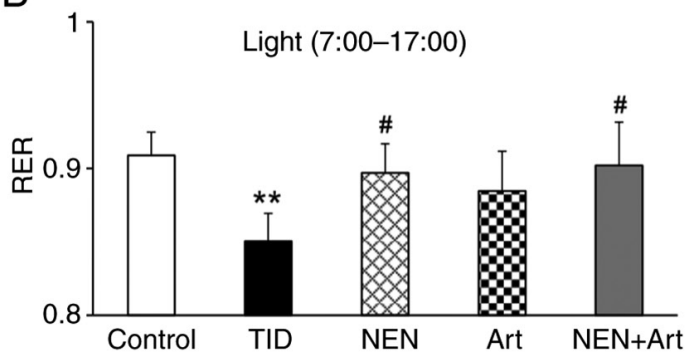

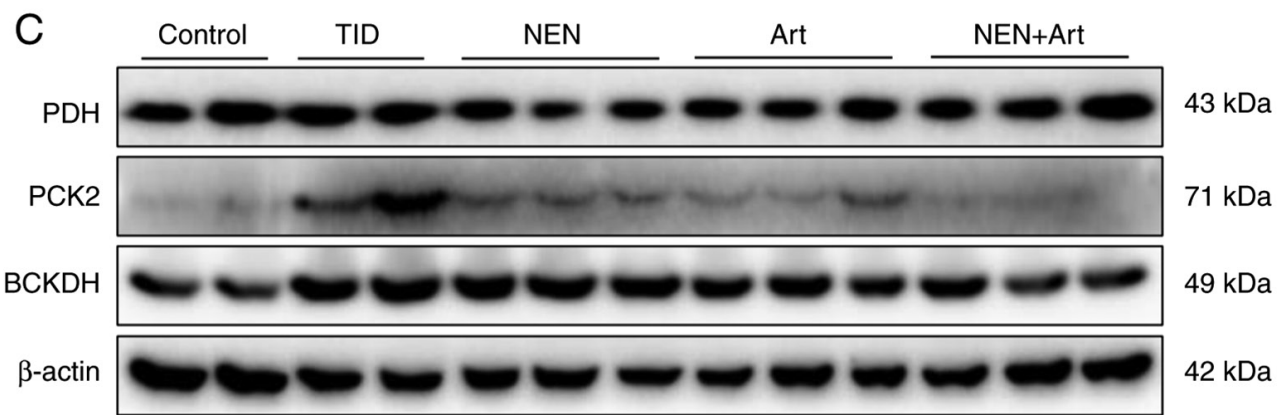
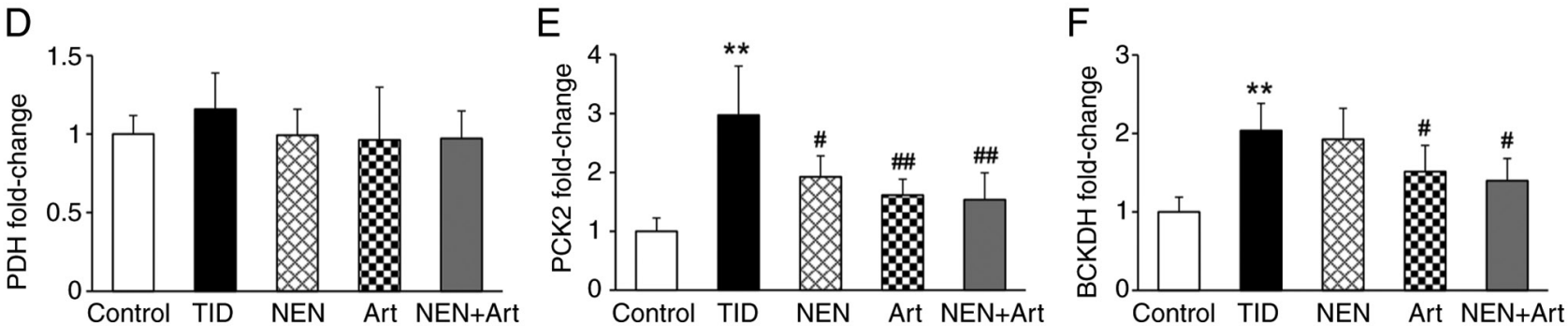

Figure 6. Effects of NEN and Art on the RER and mitochondrial metabolism. (A and B) RER values for (A) the dark and (B) light phase in each group (n=4 per group). (C-F) Western blot analysis was performed to determine the protein expression levels of PDH, PCK2, and BCKDH in the hepatic tissue of various groups. (C) Western blot bands and semi-quantitative analysis of (D) PDH, (E) PCK2 and (F) BCKDH in each group (n=4-6 per group) ${ }^{* *} \mathrm{P}<0.01$ vs. the control group; ${ }^{~} \mathrm{P}<0.05$ and ${ }^{\# /} \mathrm{P}<0.01$ vs. the T1D group. NEN, niclosamide ethanolamine salt; Art, artemether; T1D, type 1 diabetes; PDH, pyruvate dehydrogenase; PCK2, phosphoenolpyruvate carboxykinase; BCKDH, branched-chain $\alpha$-keto acid dehydrogenase complex; RER, respiratory exchange ratio.

A

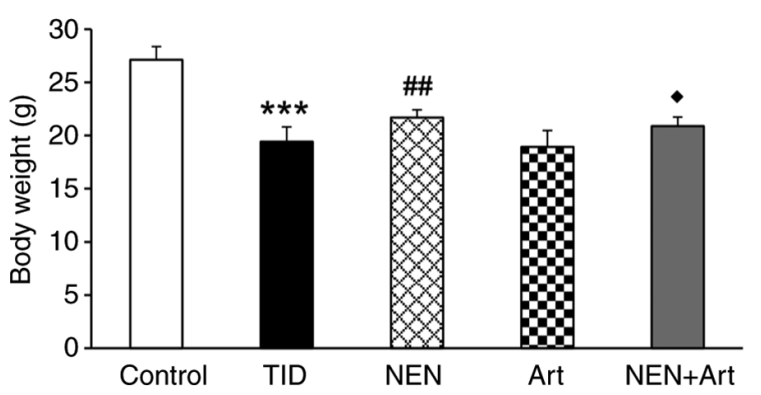

B

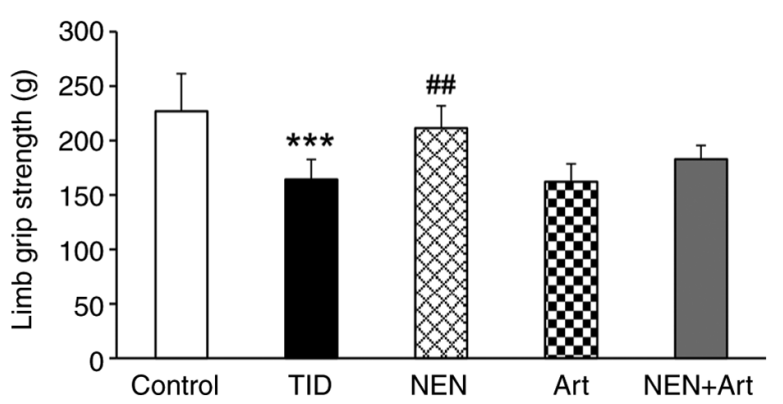

Figure 7. Effects of NEN and Art on body weight and limb grip strength. Bar graphs displaying (A) the body weight and (B) limb grip strength in each group ( $\mathrm{n}=8$ per group). ${ }^{* * *} \mathrm{P}<0.001$ vs. the control group; ${ }^{\# /} \mathrm{P}<0.01$ vs. the T1D group; ${ }^{\circ} \mathrm{P}<0.05$ vs. Art group. NEN, niclosamide ethanolamine salt; Art, artemether; T1D, type 1 diabetes.

combined treatment group. Considering that NEN and Art have different pharmacological actions $(16,17)$, it may be hypothesized that their effect may be synergistic. Hepatic metabolic disorder is characteristic in patients with T1D. Changes in various liver injury biomarkers indicated that NEN treatment alone had a hepatoprotective effect. Although Art treatment alone did not reduce abnormally elevated serum levels of ALT and AST, the liver function improved with the combination of
Art and NEN. These results indicated that NEN and Art may ameliorate liver injury via multiple signaling pathways.

Abnormal blood glucose and liver glycogen metabolism frequently occur simultaneously and restoration of one usually normalizes the other (18). Consistent with previous findings (19), a lower liver glycogen content was detected in the T1D mice. G6Pase is responsible for completing the final step in gluconeogenesis, which hydrolyzes glucose-6-phosphate to 
glucose in the endoplasmic reticulum $(20,21)$. In the present study, significantly increased hepatic G6Pase protein expression levels were observed in T1D mice. Taken together, these results suggested that augmented hepatic gluconeogenesis may contribute to impaired blood glucose and liver glycogen metabolism. The increased G6Pase protein expression levels were significantly reduced by NEN and Art alone or together to various degrees. A combination of NEN and Art also increased liver glycogen storage.

Mitochondria are the prime metabolic platform for various factors involved in energy metabolism (22). TFAM is a transcription factor that activates mitochondrial biogenesis (23). VDAC, COX IV and TOM20 are the main structural components of the mitochondria. In the present study, all these aforementioned mitochondria-related proteins were indicated to be significantly upregulated in T1D mice, which demonstrated that the hepatic mitochondrial content was increased under T1D conditions. PCK2 and BCKDH are metabolic enzymes located in the mitochondrial matrix that are associated with gluconeogenesis (24-26). Their upregulated protein expression levels in the diabetic liver in the present study suggested that the mitochondria were working harder to promote gluconeogenesis. Furthermore, the hepatic protein expression level of PDH (the key enzyme regulating pyruvate oxidation) did not increase with diabetic liver mitochondria accumulation. The RER is an important indicator to evaluate the utilization ratio of metabolic substrates (10). Diabetic T1D mice exhibited a reduced RER during both the dark and light phases of their daily cycles when compared with the control mice. The declining trend was more prominent during the dark phase. Taken together, these results indicated that mitochondrial pyruvate aerobic oxidation is relatively weakened and this is consistent with the reduction in the RER. NEN and Art ameliorated the pathological alterations of mitochondria and increased RER to various degrees. These results demonstrated that NEN and Art were able to increase the aerobic oxidation of glucose and recover the impaired mitochondrial glucose metabolism. More importantly, the effects were significantly enhanced by the combined treatment. However, the detailed mechanisms underlying the influences of NEN and Art on the RER require further investigation.

In conclusion, the present study demonstrated that combined treatment with NEN and Art improved glycometabolism and liver function more effectively than NEN or Art treatment alone in T1D mice. The underlying mechanisms may be associated with hepatic glycogen and mitochondrial metabolism. Combination therapy may be a promising approach for treating T1D in the future.

\section{Acknowledgements}

Not applicable.

\section{Funding}

This study was supported by grants from the National Natural Science Foundation of China (grant nos. 82004156 and 81673794), Shenzhen Science and Technology Project (grant no. JCYJ20190812183603627) and Shenzhen Fund for Guangdong Provincial High-level Clinical Key Specialties.

\section{Availability of data and materials}

The datasets used and/or analyzed during the current study are available from the corresponding author on reasonable request.

\section{Authors' contributions}

HS and PH designed the research. WW and HL performed most of the experiments and analyzed most of the data. ZS, PZ, $\mathrm{XY}$ and MS performed part of the experiments. $\mathrm{PH}$ and $\mathrm{HS}$ wrote the manuscript and confirmed the authenticity of all the raw data. All authors read and approved the final manuscript.

\section{Ethics approval and consent to participate}

The animal experiments in this study were approved by the Guangzhou University of Chinese Medicine Institutional Animal Care and Use Committee (Shenzhen, China).

\section{Patient consent for publication}

Not applicable.

\section{Competing interests}

The authors declare that they have no competing interests.

\section{References}

1. Warshauer JT, Bluestone JA and Anderson MS: New frontiers in the treatment of type 1 diabetes. Cell Metab 31: 46-61, 2020.

2. Guemes M, Rahman SA and Hussain K: What is a normal blood glucose? Arch Dis Child 101: 569-574, 2016.

3. Han HS, Kang G, Kim JS, Choi BH and Koo SH: Regulation of glucose metabolism from a liver-centric perspective. Exp Mol Med 48: e218, 2016.

4. Petersen MC, Vatner DF and Shulman GI: Regulation of hepatic glucose metabolism in health and disease. Nat Rev Endocrinol 13: 572-587, 2017.

5. Hu Z, Li E, Sullivan MA, Tan X, Deng B, Gilbert RG and Li C: Glycogen structure in type 1 diabetic mice: Towards understanding the origin of diabetic glycogen molecular fragility. Int J Biol Macromol 128: 665-672, 2019.

6. Marinkovic T and Oresic M: Modeling strategies to study metabolic pathways in progression to type 1 diabetes-Challenges and opportunities. Arch Biochem Biophys 589: 131-137, 2016.

7. Morio B, Panthu B, Bassot A and Rieusset J: Role of mitochondria in liver metabolic health and diseases. Cell Calcium 94: 102336, 2021.

8. Franko A, von Kleist-Retzow JC, Neschen S, Wu M, Schommers P, Böse M, Kunze A, Hartmann U, Sanchez-Lasheras C, Stoehr O, et al: Liver adapts mitochondrial function to insulin resistant and diabetic states in mice. J Hepatol 60: 816-823, 2014.

9. Wang Y, Han P, Wang M, Weng W, Zhan H, Yu X, Yuan C, Shao M and Sun H: Artemether improves type 1 diabetic kidney disease by regulating mitochondrial function. Am J Transl Res 11: 3879-3889, 2019.

10. Han P, Wang Y, Zhan H, Weng W, Yu X, Ge N, Wang W, Song G, Yi T, Li S, et al: Artemether ameliorates type 2 diabetic kidney disease by increasing mitochondrial pyruvate carrier content in $\mathrm{db} / \mathrm{db}$ mice. Am J Transl Res 11: 1389-1402, 2019.

11. Han P, Zhan H, Shao M, Wang W, Song G, Yu X, Zhang C, Ge N, Yi T, Li S and Sun H: Niclosamide ethanolamine improves kidney injury in db/db mice. Diabetes Res Clin Pract 144: 25-33, 2018.

12. Han P, Shao M, Guo L, Wang W, Song G, Yu X, Zhang C, Ge N, Yi T, Li S, et al: Niclosamide ethanolamine improves diabetes and diabetic kidney disease in mice. Am J Transl Res 10: 1071-1084, 2018.

13. Chen W, Mook RA Jr, Premont RT and Wang J: Niclosamide: Beyond an antihelminthic drug. Cell Signal 41: 89-96, 2018. 
14. Tsui KH, Wu MY, Lin LT, Wen ZH, Li YH, Chu PY and Li CJ: Disruption of mitochondrial homeostasis with artemisinin unravels anti-angiogenesis effects via auto-paracrine mechanisms. Theranostics 9: 6631-6645, 2019.

15. Carroll NV, Longley RW and Roe JH: The determination of glycogen in liver and muscle by use of anthrone reagent. J Biol Chem 220: 583-593, 1956.

16. Wang Y, Wang Y, You F and Xue J: Novel use for old drugs: The emerging role of artemisinin and its derivatives in fibrosis. Pharmacol Res 157: 104829, 2020.

17. Park JS, Lee YS, Lee DH and Bae SH: Repositioning of niclosamide ethanolamine (NEN), an anthelmintic drug, for the treatment of lipotoxicity. Free Radic Biol Med 137: 143-157, 2019.

18. Lopez-Soldado I, Guinovart JJ and Duran J: Increasing hepatic glycogen moderates the diabetic phenotype in insulin-deficient Akita mice. J Biol Chem. 296: 100498, 2021.

19. Hwang JH, Perseghin G, Rothman DL, Cline GW, Magnusson I, Petersen KF and Shulman GI: Impaired net hepatic glycogen synthesis in insulin-dependent diabetic subjects during mixed meal ingestion. A 13C nuclear magnetic resonance spectroscopy study. J Clin Invest 95: 783-787, 1995.

20. Hutton JC and O'Brien RM: Glucose-6-phosphatase catalytic subunit gene family. J Biol Chem 284: 29241-29245, 2009.
21. Lizak B, Szarka A, Kim Y, Choi KS, Németh CE, Marcolongo P, Benedetti A, Bánhegyi $G$ and Margittai É: Glucose transport and transporters in the endomembranes. Int J Mol Sci 20: 5898, 2019.

22. Chipuk JE, Mohammed JN, Gelles JD and Chen Y: Mechanistic connections between mitochondrial biology and regulated cell death. Dev Cell 56: 1221-1233, 2021.

23. Pfanner N, Warscheid B and Wiedemann N: Mitochondrial proteins: From biogenesis to functional networks. Nat Rev Mol Cell Biol 20: 267-284, 2019.

24. Yu S, Meng S, Xiang M and Ma H: Phosphoenolpyruvate carboxykinase in cell metabolism: Roles and mechanisms beyond gluconeogenesis. Mol Metab 53: 101257, 2021.

25. White PJ, McGarrah RW, Grimsrud PA, Tso SC, Yang WH, Haldeman JM, Grenier-Larouche T, An J, Lapworth AL, Astapova I, et al: The BCKDH kinase and phosphatase integrate BCAA and lipid metabolism via regulation of ATP-Citrate lyase. Cell Metab 27: 1281-1293.e7, 2018.

26. Lynch CJ and Adams SH: Branched-chain amino acids in metabolic signalling and insulin resistance. Nat Rev Endocrinol 10: 723-736, 2014. 